\title{
Pirouette Vertical Ground Reaction Force of Ballet Dancers and Non-Dancers
}

\section{CYNTHIA Y. HIRAGA ${ }^{1}$ | CAMILA SIRIANI ${ }^{1}$ | PAULO R. H. ROCHA² | DÉBORA A. DE SOUZA ${ }^{1}$ | JOSÉ A. BARELA ${ }^{1}$}

1São Paulo State University, Biosciences Institute, Campus of Rio Claro., Rio Claro, SP, Brazil

2Universidade de São Paulo, Faculdade de Medicina de Ribeirão Preto, Ribeirão Preto, SP, Brazil.

Correspondence to:Cynthia Yukiko Hiraga. Address: Avenida 24 A,1515 - Bela Vista - Rio Claro/SP, Brazil - CEP 13506-900.

email: cynthia.hiraga@unesp.br

https://doi.org/10.20338/bjmb.v14i2.159

HIGHLIGHTS
- Maximum vertical force of pirouette initial
impulse was higher in ballet dancers.
- Minimum vertical force for turning was similar
in ballet dancers and non-dancers.
- Maximum vertical force of pirouette final
impulse was similar in both groups.
- Vertical forces were similar regardless of
supporting leg dance training.
ABBREVIATIONS
ALV alleviation
ANOVAs analyses of variance
BD $\quad$ ballet dancer group
BMI $\quad$ body mass index
FI $\quad$ final impulse
ND $\quad$ no-dancer group
NPL non-preferred leg
PL $\quad$ preferred leg

HIGHLIGHTS

Quette initial

- Minimum vertical force for turning was similar

in ballet dancers and non-dancers.

- Maximum vertical force of pirouette final

impulse was similar in both groups.

- Vertical forces were similar regardless of

supporting leg dance training.

PUBLICATION DATA

Received 09122019

Accepted 15052020

Published 01072020
BACKGROUND: Different amounts of force are needed to produce an effective turn for the pirouette, especially vertical force.

AIM: To examine the vertical force produced by the supporting leg during the execution of a pirouette en dehors of ballet dancer and non-dancer participants.

METHOD: The participants included five ballet dancers who composed the ballet dancer group and eight girls without previous experience of dance training who composed the non-dancer group. The participants were invited to execute the pirouette en dehors on a force platform with each leg as the supporting leg. Two-way analyses of variance were used to test vertical reaction forces between the two groups over the preferred and non-preferred leg.

RESULTS: Among the three vertical forces measured in the present study, the maximum vertical peak for the initial impulse was significantly higher for the ballet dancers compared to the non-dancer girls. The minimum vertical force and maximum vertical peak for the final impulse were similar between both groups.

CONCLUSION: The results suggest that the initial vertical force may be critical to the pirouette en dehors, determining proficient execution of this movement in ballet dancers.

KEYWORDS: Turn | Dance | Rotation axis | Ground reaction force

\section{INTRODUCTION}

A pirouette is a complete spinning of the body on one foot, present in classical ballet choreography. This movement is widely used not only in ballet, but in other forms of dance, such as jazz, street dance, and gymnastics. According to Laws ${ }^{1}$, the pirouette constitutes a category of movement that is difficult to master. According to the author, the pirouette is a sophisticated type of motion due to a reduced support base (i.e., support on only one foot and on the tip) requiring the performer to control the forces produced with the rotating body acting on various body segments. The execution of a pirouette involves a stance position that requires activation of neuromuscular mechanisms to create a torque to initiate and maintain the turn, as well as the control of body mass distribution to determine the turn relative velocity. ${ }^{1}$

The sequence of actions of each segment for the pirouette is well described. It is characterized by one or more turns about its axis, performed on one leg in either half or full contact with the floor, while the other is bent, with the foot below the knee of the supporting leg. ${ }^{1-3}$ When the dancer starts the turn for the pirouette, he/she pushes the ground, with his/her feet, laterally in opposite directions. ${ }^{1}$ The pirouette is called en dehors when the turn is performed to the side of the raising leg (passé) and en dedans when the turn is to

\begin{tabular}{l|l|l|l|ll}
\hline Hiraga et al. & 2020 & VOL.14 & N.2 & https://doi.org/10.20338/bjmb.v14i2.159
\end{tabular}


the side of the supporting leg. ${ }^{4}$ Performance of the ballet turn, en dehors and en dedans, requires body balance provided by the movement of the leg and the arm. ${ }^{5} \mathrm{~A}$ proficient pirouette performance requires generation of torque during the turn initiation as most of the body weight is on the supporting leg in an open stance position. ${ }^{3}$

Franklin ${ }^{5}$ reported that he once observed a proficient dancer performing six or seven turns in a row easily. When analyzing the movement of this particular dancer, the author described that she created lots of torque from the very wide fourth position and performed a slight rotation of the arms and ribs over the pelvis. In addition, Franklin realized that the largest parts of the body - head, trunk, and pelvis - were well aligned over each other, and her head was spinning without inclination. A recent work ${ }^{6}$ additionally showed that experienced dancers made body adjustments in such a way as to produce a subtle base of support translations during the execution of the pirouette. Body balance is restored by a slight change in the center of mass located within the base of support. This subtle change was correlated with the number of revolutions in pirouettes. ${ }^{6}$

Some factors contribute to excellent performance of a pirouette. McMillan ${ }^{7}$ examined the execution of the pirouette in three skill levels of dancers, professionals, semi-skilled university dancers, and beginners. Kinematic profiles showed that professional dancers demonstrated larger movements and took less time in the preparation phase than semi-skilled and beginner dancers. Of interest was that the professional dancers were different from the rest of the groups in the spotting technique, arm position, right foot placement, and acceleration during the turn, while the semi-skilled dancers and beginners were not different from each other for these measures.

A strategy to maintain the control of body balance is fundamental for performing a pirouette. ${ }^{8}$ The forces acting on the floor from the feet (related to the forces exerted by the feet against the ground) result in the production of a force capable of creating conditions to start the body rotation, generating the necessary torque. An exhaustive analysis over fifty trials of pirouettes conducted by Laws ${ }^{1}$ with one professional dancer showed that all of the torques came from the two feet exerting forces for a pirouette en dehors. Pirouettes en dedans, in contrast, are initiated with a substantial torque coming from the supporting foot. The push-off foot leaves the floor early, while the supporting foot is still flat and able to exert some torque itself.

Other kinetic variables must be taken into account when performing a pirouette. Sugano and Laws ${ }^{3}$, for instance, showed, in different levels of dancers, that the pirouette improved when the width of the fourth position preparation was increased. In addition, the authors found that the initial proportion of weight on each foot must be controlled, which was best for the intermediate dancers. The initial position appears to provide a substantial contribution to a successful pirouette. A study by Lin et al. confirmed the relevance of the initial position in which professional dancers had a shorter preparation phase for the pirouette en dehors than novice dancers. It seems that novice dancers need more time to adjust their posture and plan the movement before turning. In the preparation phase of the pirouette, novice dancers applied greater push force in the posterior rather than vertical direction, than experienced dancers. ${ }^{9}$

Choosing the supporting leg indicates the preferred leg for postural stabilizing. Typically, in tasks involving both feet, such as kicking a ball, the dominant or preferred leg is the one that acts on the object or that leads the task, whereas the other leg takes the role of stabilizing body posture. ${ }^{10}$ Dance practice and training or ballet elements may influence the choice of supporting leg. For instance, a study showed the effect of Irish 
dance training on the preferred foot to stabilize posture. ${ }^{11}$ In a task that involved either foot to hop on, 53 out of the 100 non-dancer participants chose to hop on their left foot, whereas 98 of the trained dancers chose to hop on their left foot. Therefore, Irish dance training demonstrated a substantial influence on lower-limb asymmetry.

Ballet training routines are designed to use lower limbs equally. Lateral bias may occur, raising the preference for one supporting foot, as a result of years of training. This bias in pirouettes may influence functional differences between the limbs. For example, the choice of a supporting foot from the girls trained in classical dance for at least two types of pirouettes likely exploited biomechanical properties to facilitate the execution of pirouettes. ${ }^{12}$ Leg preference and leg function differences were evident according to an increase in the task demand for postural stability. ${ }^{13}$ Recently, King and Wang ${ }^{14}$ showed that functional asymmetry of the legs was more prominent in a novel goal-directed kicking task compared to a single-leg stance test.

Different kinetic aspects seem to converge in an excellent pirouette performance. The purpose of the present study is to examine the vertical forces produced by the supporting leg during a pirouette en dehors between ballet dancer and non-dancer participants. It was hypothesized that ballet dancers would show higher peak vertical forces than non-dancers throughout the course of the turn, from its initiation to its conclusion. Practice pirouettes for dance appear to enhance functional lower limb asymmetry. Thus, a higher vertical force was expected during the pirouette with the preferred leg compared with the non-preferred leg as the supporting foot.

\section{METHODS}

\section{Participants}

The participants were 13 girls aged between 15 and 25 years. Five female ballet dancers composed the ballet dancer (BD) group, with a mean age of $21.2( \pm 3.9)$, and eight girls without previous experience with any type of dance in terms of training composed the control group, non-dancer (ND) group, with a mean age of $21.2( \pm 1.2)$. The BD group was composed of girls who had been attending classical ballet classes for over ten years with a frequency of two to four times a week. The control group (i.e., ND) was composed of physically active girls from a local university. Participants from the ND group were recruited from different sport teams of the local athletic association. Participants or their guardians signed the informed consent form, approved by an Ethics Committee of the university (protocol no 9176).

\section{Procedures}

The participants were invited to the laboratory, wearing gym clothes and were weighed, barefoot in the upright posture, feet parallel, arms along the body, on a digital scale (Tanita®). The height of each participant was also measured using a stadiometer fixed to the wall. The measures of weight and height were used to calculate the body mass index (BMI) of each participant. After obtaining these measures, participants put on a pair of ballet shoes and were invited to perform the experimental task.

The experimental task consisted of performing a $360^{\circ}$ degree rotation about its axis, the pirouette en dehors, from the fifth position. It was performed over one half-pointed leg while the other was bent, with the foot below the supporting knee. ${ }^{4}$ The pirouette en 
dehors was chosen as it is the most commonly used both in ballet and in other types of dance.

Before the experimental trials, the researcher provided a video of a ballerina performing the pirouette en dehors to familiarize participants, mainly the participants without experience in classical ballet. Each participant reviewed the video at least three times to make sure that they had memorized the movement sequence. After familiarization with the idea of the movement, at least three attempts were performed on each leg (i.e., as the supporting leg) to practice the pirouette en dehors on the ground. Both groups were able to complete the task after the familiarization attempts. Naturally, the ND group performed more attempts than the BD group.

After the familiarization trials, participants were required to execute five more attempts with the right and left legs as the supporting leg on the force platform. The supporting leg chosen for the first five trials was considered to be the preferred leg. Subsequently, five more trials were performed with the other leg as supporting (i.e., the leg considered not preferred). A 30-second interval between trials and a two-minute interval between the leg conditions were allowed for each participant to avoid any fatigue effect. The participants completed the ten trials of the pirouette en dehors on a force platform (Kistler model 9286-A), which was used to acquire the ground reaction forces for the vertical and horizontal directions. These data were obtained with a frequency of $1000 \mathrm{~Hz}$ and stored for future analysis.

\section{Data Processing}

Ground reaction forces were filtered using a low-pass, second-order Butterworth digital filter, with a cut-off frequency of $20 \mathrm{~Hz}$, using a LabView routine (version 8.0, National Instruments, Austin, TX, USA).

After filtering, the ground reaction force data were normalized by the respective participant's body weight. The normalized vertical reaction force, thus, was used to obtain the initial and final impulse as the maximum vertical force peak generated to raise the center of mass at the beginning of the turn and the maximum vertical force generated to restore balance in the standing position after the turn. Finally, the alleviation of the body weight was obtained as the minimum vertical force due to the displacement of the center of mass towards the gravity force vector during the turn.

\section{Statistical Analysis}

After testing the normality and homogeneity of variance with the Shapiro-Wilk test, three analyses of variance (ANOVAs) were run, with factors group (ballet dancers $x$ nondancers) and leg (preferred and non-preferred leg), the latter treated as repeated measures. The dependent variables were initial and final impulses and the alleviation of body weight. Student's T-tests were used to compare height, weight, and BMI between groups. All statistical analyses were performed using SPSS, and the significance level was kept at 05 .

\section{RESULTS}

Table 1 presents the means and standard deviation of the anthropometric measurements of the participants for both $\mathrm{BD}$ and ND groups. Comparisons between the 
Brazilian Journal of Motor Behavior

groups did not reveal any significant differences for any of the anthropometric variables, showing that they were equivalent in terms of anthropometric measurements.

Table 1 - Means and standard deviation of height, weight, and body mass index for both BALLET DANCER (BD) and NONDANCER (ND) groups.

\begin{tabular}{lcc}
\hline & ND & BD \\
\hline Height $(\mathrm{m})$ & $1.63(0.04)$ & $1.59(0.03)^{\mathrm{ns}}$ \\
Weight $(\mathrm{Kg})$ & $54.53(3.08)$ & $51.77(4.98)^{\mathrm{ns}}$ \\
BMl $\left(\mathrm{Kg} / \mathrm{m}^{2}\right)$ & $20.5(1.00)$ & $20.2(1.76)^{\mathrm{ns}}$ \\
\hline
\end{tabular}

ns $p$-values $>.05$

Figure 1 depicts the initial impulse for both BD and ND groups obtained for both preferred and non-preferred legs. Fz is the vertical component of the ground reaction force. ANOVA revealed a significant effect for group, $F(1,11)=7.37, p<.05, \eta 2=.40$; but no effect for leg, $F(1,11)=.00, p>.05, \eta 2=.00$; and no group and leg interaction, $F(1,11)$ $=.00, p>.05, \eta 2=.01$. The mean maximum vertical force for the initial impulse was higher for the BD compared to the ND participants.

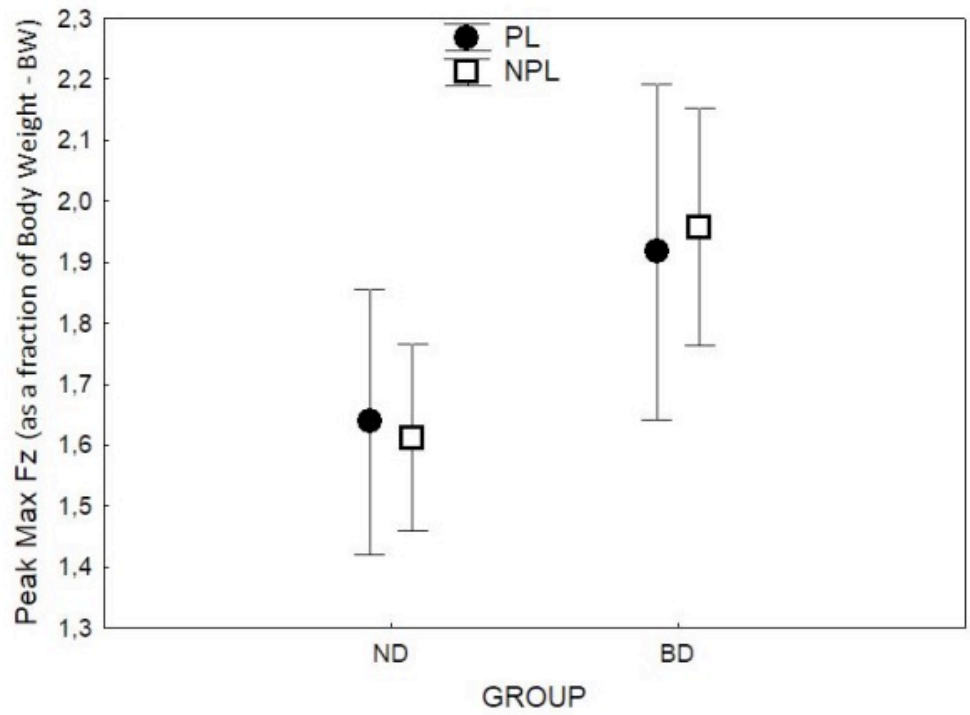

Figure 1.Mean and standard deviation of maximum vertical force peak of the initial impulse for both nondancer (ND) and ballet dancer (BD) groups obtained for the preferred leg (PL) and non-preferred leg (NPL).

Table 2 shows mean and standard deviation for the alleviation (i.e., minimum vertical force during the turn) and maximum vertical force for the final impulse for both $B D$ and ND groups, obtained for both PL and NPL. For the alleviation, ANOVA did not reveal any effect for group, $F(1,11)=2.10, p>.05, \eta 2=.16$; leg, $F(1,11)=0.58, p>.05, \eta 2$ $=.05$; or group and leg interaction, $F(1,11)=0.50, p>.05, \eta 2=.04$. Similarly, for the final impulse, ANOVA did not reveal any effect for group, $F(1,11)=.14, p>.05, \eta 2=.01$; leg, $F$ $(1,11)=1.48, p>.05, \eta 2=.11$; or group and leg interaction, $F(1,11)=.00, p>.05, \eta 2$ $=.00$. 
Table 2 - Means and standard deviation of the alleviation (ALV) and final impulse (FI) for both non-dancer (ND) and ballet dancer (BD) groups and the preferred leg (PL) and non-preferred leg (NPL).

\begin{tabular}{lcccc}
\hline & \multicolumn{2}{c}{ ND } & \multicolumn{2}{c}{ BD } \\
\hline ALV & PL & NPL & PL & NPL \\
FI & $0.50(0.24)$ & $0.51(0.20)$ & $0.31(0.21)$ & $0.40(0.12)$ \\
\hline
\end{tabular}

\section{DISCUSSION}

The present study aimed to examine the kinetic profile of the pirouette movement from ballet dancer and non-dancer participants. The focus was to investigate the production of vertical force with the preferred and non-preferred leg as a support leg. Our results showed that although ballet dancers produce more force at the beginning of the pirouette en dehors, after this initial higher impulse, the management of the applied force does not differ throughout the execution of the movement. The force applied to the ground to initiate the movement (i.e., the initial impulse) appears to be relevant to the execution of the pirouette en dehors.

The positions of the feet in ballet are a fundamental part of the ballet technique, defining standard feet placements on the floor to begin a pirouette. The higher vertical force peak observed in the present study for the ballet dancers compared to the nondancer participants could be related to a better fifth position to initiate the turn, since it is a more challenging technique. In contrast, Lin et al. ${ }^{9}$ found no differences in vertical force direction of the execution of pirouettes between experienced and novice ballet dancers with feet in the fourth position. Either placement of feet on the floor or some experience in ballet (i.e., non-dancers vs. novice ballet dancers) might determine the production of vertical force to initiate the turn.

Interestingly, Sugano and Laws ${ }^{3}$ showed that a wider preparation position was determinant for successful pirouettes, observed for experienced ballerinas. In the case of the participants of the current study, the substantial amount of experience, at least ten years, enables them to properly use their feet in the fifth position to initiate the turn. The greatest initial impulse generated by experienced dancers possibly moved their center of mass upward to a point so that they can efficiently perform or rotate fully $\left(360^{\circ}\right) .{ }^{1}$ This force, according to Laws ${ }^{1}$, may be decisive for excellent performance in the current study, confirmed by the researcher through visual inspection. In the present study, kinematic data or qualitative assessment were not collected. The visual inspection relied on the knowledge and experience of one of the researchers who is a ballerina.

Higher vertical forces would be necessary to gain greater height during the flight. Taking into account that balance and weight shifting are essential elements in ballet, ${ }^{8}$ the production of forces permits sufficient time to perform the necessary body rotation in the air. However, the necessity to reach higher heights does not only demand higher force, but also that the force is applied in a shorter time in order for a larger impulse and faster speed at take-off. These mechanical requirements are even trickier for the non-dancers because of the required balance to push off and gain the necessary momentum to pivot the body in a short time, as well as a proper landing in a graceful position without losing balance. 
The standard deviation of the vertical force peak was also higher for the ballet dancers compared with the non-dancers. Technical performance, where the ballet elements are executed repeatedly with stability and little variation, reflects the results of long-term training and a great deal of practice. However, the skill acquisition process is associated with the dynamic variability of movement practices that facilitates learning in biological systems. ${ }^{15}$ Although it was not possible to demonstrate this process in the current study, the variability in motor behavior emerges over time, and can be captured by nonlinear tools. A recent work showed that the results of entropy in the sample indicated more irregular characteristics of postural sway in ballet dancers, representing more automated postural control. ${ }^{16}$

Interestingly, poor performance in novice dancers is suggested to occur due to, among other factors, an insufficient proximal-to-distal extension sequence pattern and a higher push force in propulsion in mediolateral, but not vertical and anterior-posterior directions. ${ }^{9}$ These suggested characteristics leading to unskilled performance would contribute to lower initial impulse, as observed in the current study. Moreover, Walter and colleagues ${ }^{17}$ found higher vertical peak during a single pirouette in pointe shoes compared with flat shoes in experienced dancers. For the authors, the pointe shoes might cause an increase in upward movements of the center of mass position or by the more rigid outsole of pointe shoes compared with flat shoes. In all these cases, there are indications that the initial force production, moving the center of mass upward at the beginning of the pirouette, is critical, and our data showed that this issue is a differential between ballet dancer and non-dancer participants.

Although the initial peak force is a differential aspect characterizing ballet dancer and non-dancer participants during the pirouette, other components of the vertical force do not play any role in pirouette performance. The vertical force during the alleviation phase and in the final impulse to conclude the turn did not differ between groups. Both ballet dancers and non-dancer girls produced a similar amount of vertical ground forces during the pirouette. These results do not minimize or exclude the relevance of these forces for the pirouette performance. Instead, the results indicate that despite the experience of the performer, the required level of force production is attained to perform the pirouette.

Finally, the results showed no differences in any of the forces examined between the preferred or non-preferred supporting leg, regardless of group. Lin et al.18 demonstrated that experienced dancers performed better pirouettes when they used their preferred leg as the supporting leg to start the turn. Specifically, the trunk rotation axis assumed a more upright trunk posture when compared to the non-preferred supporting leg. The authors ${ }^{18}$ reported that this difference in performance did not occur for the novice ballerina group. It is possible that variables related to kinetics, in the case of a pirouette, may be narrower to distinguish performance differences between supporting legs to initiate the turn. An alternative explanation may be due to the level of experience of the dancers in the present study. Although the participants had at least ten years of practice, they started at an early age and, therefore, had not had time to become ballerinas at professional levels.

The pirouette movement is complex and provides a critical window to further understand some learning aspects related to overall motor behavior. We hope that the current study contributes to fill this gap. Moreover, we would like to acknowledge the two limitations of this study. First is the broad age range of the participants that varied from 15 to 25 years. The broad age range issue might contribute to developmental bias in the 
overall results. It was assumed that establishing ten years of practice or training for the ballet dancer group would reduce the effect of developmental bias and sample heterogeneity. Secondly, additional measurements associated with kinetics might explain some of the results and provide understanding of how expert dancers combine the generation of muscular force, joint torque, and activation of intra- and inter-limb coordination to perform the pirouette. Future investigations related to these variables may reveal new discoveries.

\section{CONCLUSION}

The results of the present study indicated a significant difference between ballet dancer and non-dancer participants in the initial impulse for the pirouette performance. The initial impulse reflects the vertical force peak in the first stage to perform the pirouette en dehors. This is probably because many years of ballet training enabled the dancers to take advantage of the initial strength needed to provide sequence and resume the movement with adequate balance. This suggests that the initial vertical force may be essential to a well performed pirouette en dehors. We hope that the findings of the current study will provide dance educators with more details about movement turning to improve teaching and to help novice dancers to learn the turning movement with relevant cues to facilitate learning complex turns.

\section{REFERENCES}

1. Laws KL. An analysis of turns in dance. Dance Res J. 1978;11:12-19. doi: https://doi.org/10.2307/1477841.

2. Biringen EK. Analysis of pirouette execution for improved performance. Med Probl Perform Ar. 2010;25:136-137.

3. Sugano A, Laws K. Physical analysis as a foundation for pirouette training. Med Probl Perform Ar. 2002;17:29-32.

4. Faro JA, Sampaio LP. Dicionário de Balé e Dança. Rio de Janeiro, RJ: Jorge Zahar; 1989.

5. Franklin EN. Dance Imagery for Technique and Performance. Champaign, IL: Human Kinetics; 1996.

6. Lott MB. Translating the base of support: a mechanism for balance maintenance during rotations in dance. J Dance Med Sci. 2019;23:17-25. doi: https://doi.org/10.12678/1089313x.23.1.17.

7. Krasnow D, Wilmerding M, Stecyk S, Wyon M, Koutedakis Y. Biomechanical research in dance: a literature review. Med Probl Perform Ar. 2011;26:3-23.

8. Pedersen C, Erleben K, Sporring J. Ballet balance strategies. Simul Model Pract Theory. 2006;14:1135-1142. doi: https://doi.org/10.1016/j.simpat.2006.09.009.

9. Lin CW, Chen SJ, Su FC, Wu HW, Lin CF. Differences of ballet turns (pirouette) performance between experienced and novice ballet dancers. Res Q Exerc Sport. 2014;85:330-340. doi: https://doi.org/10.1080/02701367.2014.930088. 
Brazilian Journal of Motor Behavior

10. Gabbard C, Iteya M. Foot laterality in children, adolescents, and adults. Laterality. 1996;1:199-205. doi: https://doi.org/10.1080/713754236.

11. Cromie S, Greenwood JG, McCullagh JF. Does Irish-dance training influence lower-limb asymmetry? Laterality. 2007;12:500-506. doi: https://doi.org/10.1080/13576500701575140.

12. Golomer E, Rosey F, Dizac H, Mertz C, Fagard J. The influence of classical dance training on preferred supporting leg and whole body turning bias. Laterality. 2009;14:165-177. doi: https://doi.org/10.1080/13576500802334934.

13. Wang Z, Newell KM. Footedness exploited as a function of postural task asymmetry. Laterality. 2013;18:303-318. doi: https://doi.org/10.1080/1357650x.2012.672423.

14. King AC, Wang Z. Asymmetrical stabilization and mobilization exploited during static single leg stance and goal directed kicking. Hum Movement Sci. 2017;54:182-190. doi: https://doi.org/10.1016/j.humov.2017.05.004.

15. Stergiou N, Decker LM. Human movement variability, nonlinear dynamics, and pathology: Is there a connection? Hum Movement Sci. 2011;30:869-888. doi: https://doi.org/10.1016/j.humov.2011.06.002.

16. Michalska J, Kamieniar A, Fredyk A, Bacik B, Juras G, Slomka KJ. Effect of expertise in ballet dance on static and functional balance. Gait Posture. 2018;64:68-74. doi: https://doi.org/10.1016/j.gaitpost.2018.05.034.

17. Walter H, Docherty C, Schrader J. Ground reaction forces in ballet dancers landing in flat shoes versus pointe shoes. J Dance Med Sci. 2011;15:61-64.

18. Lin CW, Su FC, Wu HW, Lin CF. Effects of leg dominance on performance of ballet turns (pirouettes) by experienced and novice dancers. J Sport Sci. 2013;31:1781-1788. doi: https://doi.org/10.1080/02640414.2013.803585.

Citation: Hiraga CY, Siriani C, Rocha RH, de Souza DA, Barela JA.Pirouette Vertical Ground Reaction Force of Ballet Dancers and Non-dancers. BJMB. 2020: 14(2): 53-61.

Editors: Dr Fabio Augusto Barbieri - São Paulo State University (UNESP), Bauru, SP, Brazil; Dr Natalia Madalena Rinaldi - Federal University of Espírito Santo (UFES), Vitória, ES, Brazil.

Copyright:@ 2020 Hiraga, Siriani, Rocha, de Souza and Barela and BJMB. This is an open-access article distributed under the terms of the Creative Commons Attribution-NonCommercial-NoDerivatives 4.0 International License which permits unrestricted use, distribution, and reproduction in any medium, provided the original author and source are credited.

Funding: This research did not receive any specific grant from funding agencies in the public, commercial, or not-forprofit sectors.

Competing interests: The authors have declared that no competing interests exist.

DOl:https://doi.org/10.20338/bjmb.v14i2.159 